\title{
Measurement of interstation phase velocity by wavelet transformation*
}

\author{
Qingju $\mathrm{Wu}^{\star} \quad$ Xiufen Zheng Jiatie Pan Fengxue Zhang and Guangcheng Zhang \\ Institute of Geophysics, China Earthquake Administration, Beijing 100081, China
}

\begin{abstract}
In this paper, we present wavelet transformation method to measure interstation phase velocity. We use Morlet wavelet function as mother wavelet to filter two seismograms at various period of interest, and correlate the wavelet filtered seismograms to form cross-correlogram. If both wavelet filtered signals are in phase at that period, the phase of the cross-correlogram is a minimum. Using 3-spline interpolation to transform cross-correlation matrix to a phase velocity verse period image, it is convenient for us to measure interstation phase velocity.
\end{abstract}

Key words: dispesion, phase velocity, wavelet transform

CLC number: P315.63 Document code: A

\section{Introduction}

Analysis of surface wave dispersion information is a useful means to determine crust and upper mantle structure, this information include group arrival time, phase angle, and amplitude as a function of period. Since its introduction by Dziewonski et al (1969), frequency-time analysis has been widely used in seismology to measure surface wave dispersion. Determinations of surface wave dispersion have commonly done using either single-station or two-station methods, a number of different approaches have been developed for the measurement of group and phase velocities and attenuation (Kovach, 1978; Nakanishi, 1979; Taylor and Toksoz, 1982; Hwang and Mitchell, 1986).

Single-station methods assume that either the initial phase of the earthquake source is known or its effects are small enough to be ignored when they are used to determine surface wave dispersion. However, Knopoff and Schwab (1968) have shown that corrections to these measurements are necessary whenever the seismic source is not oriented in either a purely horizontal or a purely vertical direction.

Two-station methods avoid the necessity of knowing the earthquake source information, but they require

\footnotetext{
* Received 2 June 2009; accepted in revised form 12 July 2009; published 10 August 2009.

^Corresponding author. e-mail: wuqj@cea-igp.ac.cn
}

that the two stations should lie on a common great circle path with the earthquakes. Bloch and Hales (1968) provided to pass both seismograms through a narrow bandpass digital filter and form cross-correlogram to measure interstation phase velocity. Landisman et al (1969) noticed that considerable reduction of the noise level and stabilization of measured phase velocity can be achieved by windowing a cross-correlogram, since the cross -correlation is an approximation of the interstation impulse response. Narrow band-pass cross-correlogram is a commonly used method to determine interstation phase velocity. A different approach to the problem of calculating the interstation phase velocity have been given by Taylor and Toksoz (1982) and Hwang and Mitchell (1986), which are based on the use of time domain and a frequency domain Wiener filters respectively.

Either single-station method or two-station method is implemented by frequency-time analysis, Gabor-Heisenberg inequality demonstrates that there is a trade-off between time resolution and frequency resolution. In order to give frequency and time information equal accuracy for phase velocity measurement, we present wavelet transformation method to determine interstation phase velocity.

In this paper, we firstly summarize the commonly used method to measure interstation phase velocity, then present wavelet analysis method, and give some synthetic and real data examples to show the application. 


\section{Interstation phasevelocity measurement}

Interstation phase velocity is calculated from the phase spectra of Green functions using the formula

$$
c(f)=\frac{f \Delta}{f t_{0}+[\varphi(f) \pm N]},
$$

where $f$ is the frequency, $\Delta$ is the interstation distance, $t_{0}$ is the first time point of the Green function, and $\varphi$ is the phase of the Green function in cycles, $N$ is an integer.

In order to reduce noise and multimode interference, and stabilize phase velocity measurement, narrow-band pass cross-correlogram is extensively used to measure interstation phase velocity. This method is to pass both seismograms through a narrow bandpass digital filter centered at group-delay time corresponding to the various periods of interest and form the cross product of the filtered seismograms, after time shifting. The average of the resultant time series is a maximum when the two signals are in phase. This process is approximately equivalent to time-variant filtering in that the limited portion of the seismograms used will eliminate the unwanted noise and non-least time path arrivals outside each window.

A digital bandpass boxcar shaped filter is commonly used to do narrow band pass filtering, given by

$$
b_{t}=\frac{\sin 2 \pi h t}{\pi t} \cos 2 \pi f_{0} t,
$$

where $2 h$ is the bandwidth and $f_{0}$ is the center frequency.

Because $b_{t}$ is symmetric, the filter introduces no phase shift. The practical situation requires that the length of the digital filter be finite, windowing should be applied to the infinite filter to produce finite digital filter, instead of merely truncating. The coefficients of the finite digital filter is usually taken to be

$$
\begin{gathered}
C_{n}=\frac{\sin (2 \pi h n \mathrm{~d} t)}{\pi n \Delta t} . \\
\cos \left(2 \pi f_{0} n \mathrm{~d} t\right) \cos \left(\frac{\pi n}{2 N}\right) .
\end{gathered}
$$

Where $\mathrm{d} t$ is the sampling rate, both $n$ and $N$ are positive integer. Noted from the above formula, a cosin window is used to windowing data.

\section{Wavelet transform}

Wavelet analysis, also known as multi-resolution analysis, uses a series of dilated and delayed oscillatory functions to decompose a time-varying signal into its nonstationary spectral components. There are two key advantages of wavelet analysis over traditional Fouries analysis and windowed Fourier methods, one is that the wavelet analysis retains information on how the spectral content varies with time delay, and another is that the accuracy of the time and frequency remain constant over the entire time-frequency domain.

We present wavelet analysis to measure interstation phase velocity. In our method, we use a non-orthonormal Morlet wavelet $g(t)$, composed of a harmonic wave modulated by a Gaussian envelope, as mother function

$$
g(t)=\exp \left(\frac{-t^{2}}{2 \sigma^{2}}\right) \exp \left(\frac{\mathrm{i} 2 \pi t}{T_{0}}\right),
$$

where $T_{0}$ is the central period.

The mother wavelet is dilated and delayed to produce a set of daughter wavelet according to

$$
g\left(\frac{t-\tau}{\alpha}\right)=\exp \left[\frac{-(t-\tau)^{2}}{2(\sigma \alpha)^{2}}\right] \exp \left[\frac{\mathrm{i} 2 \pi(t-\tau)}{\alpha T_{0}}\right],
$$

where $\alpha$ is the dilating scale, and $\tau$ is the time delay.

The central frequency of the daughter wavelet is thus $\alpha / T_{0}$. The wavelet transform of a signal is obtained by integrating the time varying signal $S(t)$ over the daughter wavelet as a function of dilating scale $\alpha$ and time delay $\tau$.

$$
W(\alpha, \tau)=\frac{1}{\sqrt{\alpha}} \int_{-\infty}^{\infty} g^{*}\left(\frac{t-\tau}{\alpha}\right) S(t) \mathrm{d} t,
$$

where $g^{*}$ is the complex conjugate of the daughter wavelet, the resulting signal is complex, with both amplitude and phase information.

Actually, the transformed signal is the stacking results within a certain time-frequency subdomain centered at central frequency and delay time.

We use equation (6) to do wavelet transformation for the two seismogram located at the great circle with the earthquake, each period of interest is determined by the central frequency of the daughter wavelet, we correlate the two wavelet transformed signals at each period to get a cross-correlogram. If both wavelet filtered signals are in phase at that period, the phase of the cross-correlogram is a minimum. Thus, wavelet transform provides us a convenient method to measure interstation phase velocity, particularly, the resulting cross-correlogram retain equal time-frequency accuracy in the time-frequency domain. In order to directly show the relationship between periods and phase velocities, we 
use 3-spline interpolation to transform cross-correlation matrix to a phase velocity verse period image.

\section{Synthetic data test}

We use synthetic seismogram to test wavelet analysis method. Synthetic Rayleigh seismograms are generated at distance of 1000 and $2000 \mathrm{~km}$ from the sources. The model used to generate synthetic Rayleigh
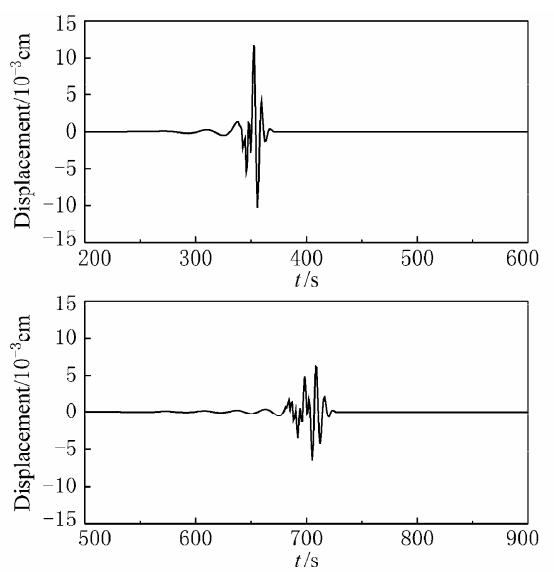

(a)

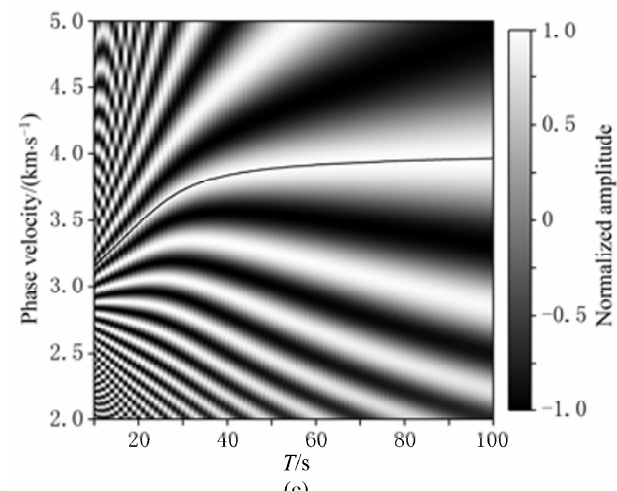

(c) seismograms is listed in Table 1. The seismograms are shown in Figure 1a. The theoretical phase velocity is shown in Figure 1b.

Table 1 Model parameters for synthetic Rayleigh waves

\begin{tabular}{cccc}
\hline Thickness $/ \mathrm{km}$ & $v_{\mathrm{P}} /\left(\mathrm{km} \cdot \mathrm{s}^{-1}\right)$ & $v_{\mathrm{S}} /\left(\mathrm{km} \cdot \mathrm{s}^{-1}\right)$ & Density $/\left(\mathrm{g} \cdot \mathrm{cm}^{-3}\right)$ \\
\hline 10.0 & 5.52 & 3.20 & 2.56 \\
10.0 & 6.08 & 3.50 & 2.72 \\
20.0 & 6.65 & 3.80 & 2.88 \\
$\infty$ & 8.10 & 4.40 & 3.36 \\
\hline
\end{tabular}

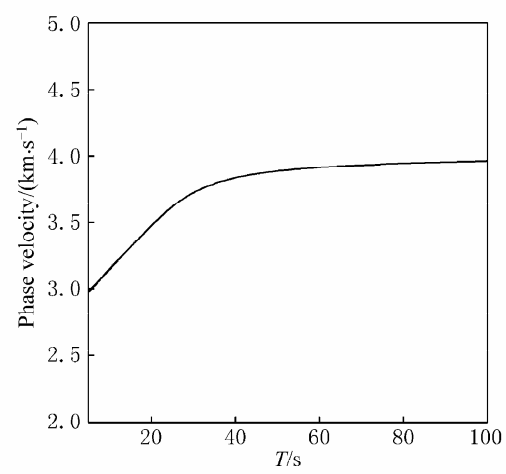

(b)

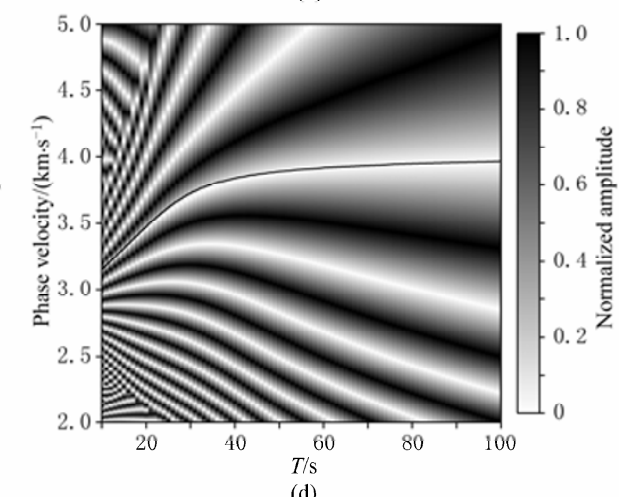

Figure 1 (a) The synthetical Rayleigh wave seismograms; (b) The theoretical phases velocity; (c) The interstation phase velocity measured by traditional narrow band pass corss-correlogram; (d) The interstation phase velocity measured by wavelet analysis.

We use both narrow band pass cross-correlogram and wavelet analysis to calculate the interstation phase velocity and the results are shown in Figures 1c and 1d, respective, the theoretical phase velocity are also overlapped on the two transform image. For narrow band pass cross-correlogram, the image represents the normalized correlation coefficient for the two narrow band passs signal centred at each period, the value is within $[-1.0,1.0]$. Thus, the coordinates of the maximum values located among physical phase velocity range is taken to be the phase velocity samples. The resolution is determined by the width where the maximum normalized correlation coefficient is damped to a given level, similar to the pass band width definition. For wavelet cross-correlogram, the image represents the absolute phase for the complex cross-correlogram between two wavelet transformed signals centred at each period, normalized by $\pi$, the value is range from 0.0 to 1.0 . Thus, the coordinates of the minimum values located among physical phase velocity range is taken to be the 
phase velocity samples. The resolution can be determined by the width where the normalized minimum phase is increased to a given level. Anyway, the resolution is easy to be detected from the colored images. We note that wavelet analysis shows better resolution than traditional narrow band pass analysis, and it delineate that wavelet analysis is a good method to measure interstation phase velocity.

\section{Application for real data}

We use both narrow band pass correlation and wavelet filtered correlation to measure phase velocity of real surface wave data. We take a short surface wave path and a long surface wave path covering the North China and the South China, respectively, the response band is up to $60 \mathrm{~s}$ and $120 \mathrm{~s}$ for the short and long path
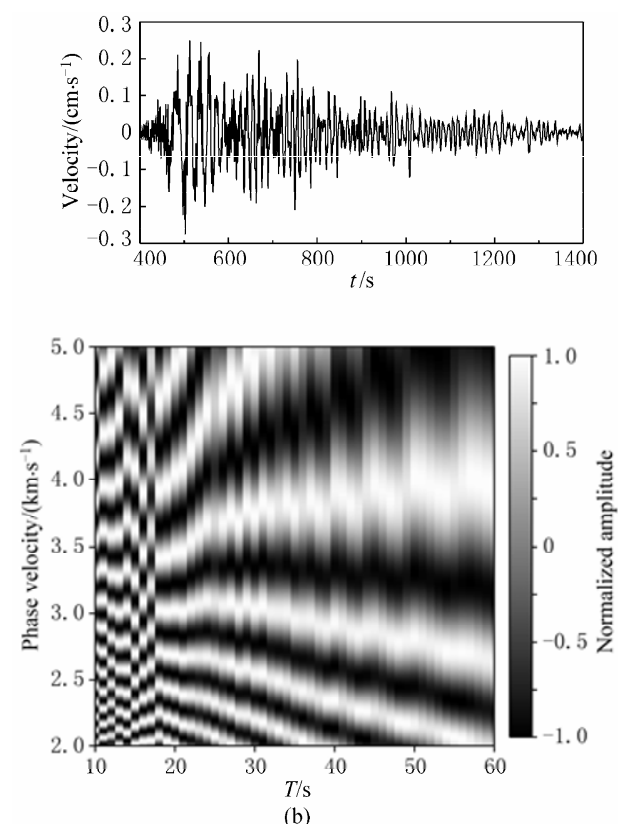

Figure 2 (a) The real data for Rayleigh wave seismogram; (b) The interstation phase velocity measured by traditional narrow band pass corss-correlogram; (c) The interstation phase velocity measured by wavelet analysis.

We also use another $M_{\mathrm{S}} 6.1$ event located at $\left(52.33^{\circ} \mathrm{N}, 173.95^{\circ} \mathrm{E}\right)$ with event depth of $45.0 \mathrm{~km}$, crossing the South China between two stations with epicentral distance at 5704.4 and $6576.5 \mathrm{~km}$, respectively, and the azimuthal difference between the near-source and the far-source station is $3.4^{\circ}$. Figure $3 \mathrm{a}$ shows the vertical component seismogram recorded at two stations. The interstation phase velocity measured by wavelet analysis and traditional narrow band methods is shown in Figures $3 b$ and $3 c$, respectively. Com-

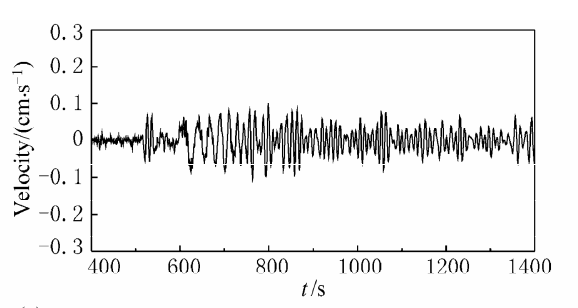

(a)

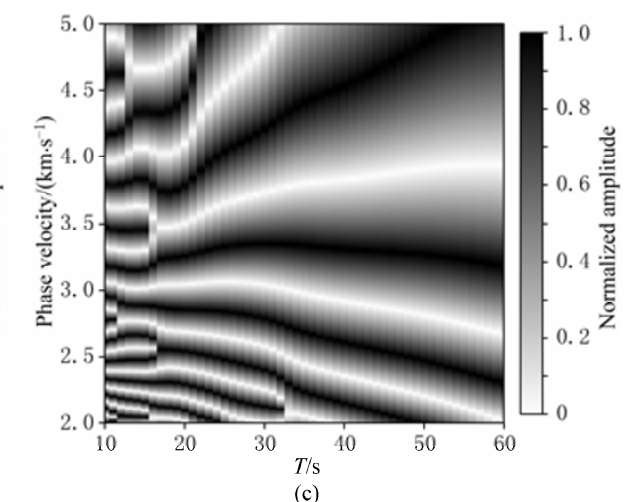

(c)

respectively.

The proposed technique is firstly applied to a surface wave path from an $M_{\mathrm{S}} 6.7$ event occurred at $\left(37.32^{\circ} \mathrm{N}, 136.62^{\circ} \mathrm{E}\right)$, with event depth of $5.0 \mathrm{~km}$, crossing the North China between two stations with epicentral distance at $1576.7 \mathrm{~km}$ and $2052.2 \mathrm{~km}$ respectively, and the azimuthal difference between the near-source and the far-source station is $3.4^{\circ}$. Figure $2 \mathrm{a}$ shows the vertical component seismogram recorded at two stations. The interstation phase velocity measured by wavelet analysis and traditional narrow band methods is shown in Figures $2 \mathrm{~b}$ and 2c, respectively. Compared to the two images, it is obvious that wavelet transform method is advantage with higher resolution over traditional methods.

pared to the two images, it is obvious that wavelet transform method provide a better phase-velocity verse period image than narrow pass band correlation method.

\section{Conclusions}

In this paper, we have applied a wavelet transform approach to the problem of determining the interstation phase velocity from surface wave. Wavelet transform can provide a time-frequency image which represents 

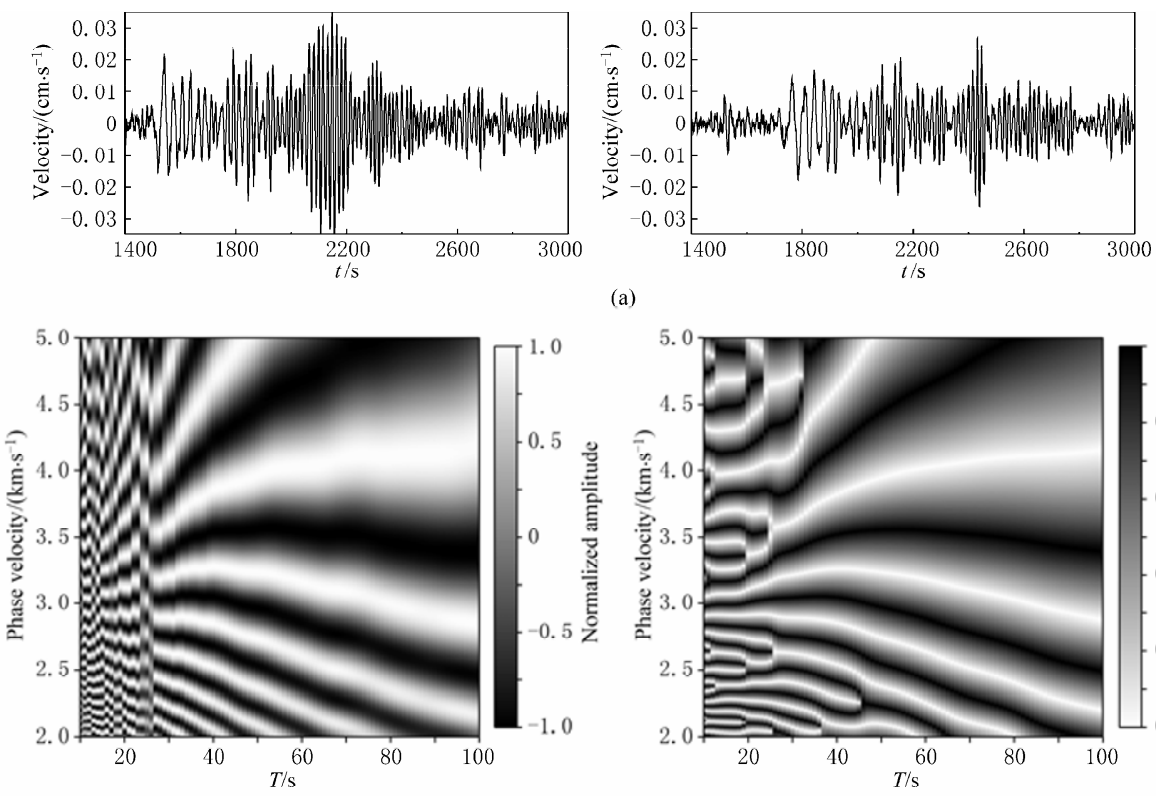

(a)

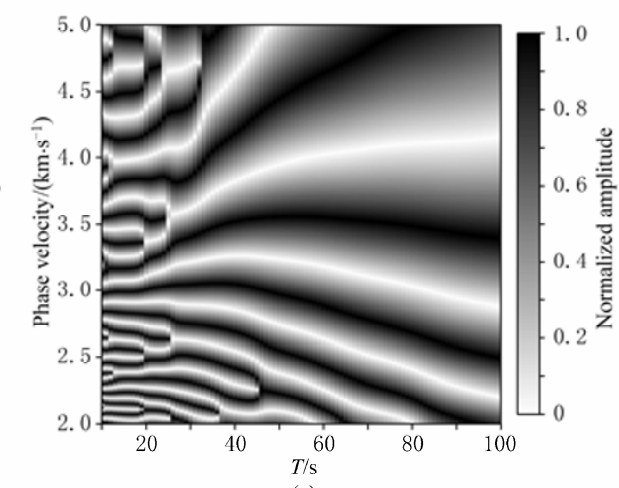

(c)

Figure 3 (a) The real data for Rayleigh wave seismogram; (b) The interstation phase velocity measured by traditional narrow band pass corss-correlogram; (c) The interstation phase velocity measured by wavelet analysis.

the time variable spectrum localized at each individual time, such a image can be mapped to period-phase velocity domain, and phase velocity is picked to the point with minimum phase. Compared to narrow band pass filtering and correlation method using a fixed-length filter to bandpass data at every period, wavelet transform use a variable length filter to bandpass data for each period, i.e., the shorter period, the shorter length, the longer period, the longer length, to obtain spectrum with different width, i.e., the longer period, the smaller width, the short period, the larger width. The trade-off between time and frequency resolution is automatically adaptive by the dilating scale of wavelet, thus, wavelet analysis provide equal time and frequency accuracy. Tests conducted using synthetic and real data showed that the wavelet technique give results with high resolution than traditional methods, and can be used to measure interstation phase velocity.
Acknowledgements This work is funded by National Natural Science Foundation of China (No.40774039). We are grateful to two anonymous reviewers and the editor for their suggestions to improve our manuscript. We also thank the Seismic Data Backup Center of China Seismic Netwrok.

\section{References}

Bloch S and Hales A L (1968). New techniques for the determination of surface wave phase velocities. Bull Seism Soc Amer 58: 1 021-1 034.

Dziewonski A, Bloch S and Landisman N (1969). A technique for the analysis of transient seismic signals. Bull Seism Soc Amer 59: 427-444.

Hwang H J and Mitchell B J (1986). Interstation surface wave analysis by frequency-domain Wiener deconvolution and modal isolation. Bull Seism Soc Amer 76: 847-864.

Landisman M, Dziewonski and Sato Y (1969). Recent improvements in the analysis of surface wave observations. Geophys $J R$ astr Soc 17: 369-401.

Nakanishi I (1979). Phase velocity and $Q$ of mantle Rayleigh waves. Geophys $J R$ Soc 58: 35-59.

Knopoff L and Schwab F A (1968). Apparent initial phase of a source of Rayleigh waves. $J$ Geophys Res 73: 755-760.

Kovach R L (1978). Seismic surface waves and crustal and upper mantle structure. Rev Geophys Space Phys 16: 1-13.

Taylor S R and Toksoz M N (1982). Measurement of interstation phase and group velocities and $Q$ using Wiener filtering. Bull Seism Soc Amer 72: 73-91. 